\title{
Review of: "A life cycle assessment of reprocessing face masks during the Covid-19 pandemic"
}

\section{Mohammad Sharifi ${ }^{1}$}

1 University of Tehran

Potential competing interests: The author(s) declared that no potential competing interests exist.

Dear Gabriele Marinello,

Hope you are good. Valuable research has been done. The research results are useful. 